\title{
A New Class of Bianchi Type-I Cosmological Models in Scalar-Tensor Theory of Gravitation and Late Time Acceleration
}

\author{
Anirudh Pradhan ${ }^{1}$, Ajay Kumar Singh ${ }^{2}$, H. Amirhashchi ${ }^{3}$ \\ 1,2 Department of Mathematics, Hindu Post-graduate College, Zamania-232 331, Ghazipur, India \\ 1e-mail: pradhan@iucaa.ernet.in; pradhan.anirudh@gmail.com \\ ${ }^{3}$ Young Researchers Club, Mahshahr Branch, Islamic Azad University, Mahshahr, Iran \\ e-mail: h.amirhashchi@mahshahriau.ac.ir
}

\begin{abstract}
A new class of a spatially homogeneous and anisotropic Bianchi type-I cosmological models of the universe for perfect fluid distribution within the framework of scalar-tensor theory of gravitation proposed by Sáez and Ballester (Phys. Lett. 113:467, 1986) is investigated. To prevail the deterministic solutions we choose the different scale factors which yield time-dependent deceleration parameters (DP) representing models which generate a transition of the universe from the early decelerated phase to the recent accelerating phase. Three different physically viable models of the universe are obtained in which their anisotropic solutions may enter to some isotropic inflationary era. The modified Einstein's field equations are solved exactly and the models are found to be in good concordance with recent observations. Some physical and geometric properties of the models are also discussed.
\end{abstract}

Key words: Bianchi type-I universe, Exact solution, Alternative gravitation theory, Variable deceleration parameter

PACS: 98.80.-k

\section{Introduction}

General relativity (GR) passes all present tests with flying colours, however, there are several reasons why it remains very important to consider alternative theories of gravity. The first one is that theoretical attempts at quantizing gravity or unifying it with other interactions generically predict deviation from Einstein's theory, because gravitation is no longer mediated by a pure spin-2 field but also by partners to the graviton. The second reason is that it is any way extremely instructive to contrast GR's predictions with those of alternative models, even if there were no serious theoretical motivation for them. The third reason is the existence of several puzzling experimental issues, which do not contradict GR in a direct way, but may nevertheless suggest that gravity does not behave at large distances exactly as Newton and Einstein predicted. Cosmological observations notably tell us that about $96 \%$ of the total energy density of the universe is composed of unknown, non-baryonic, fluids ( $72 \%$ of "dark energy" and $24 \%$ of "dark matter"), and the acceleration of the two Pioneer spacecrafts towards the Sun happens to be larger than what is expected from the $1 / r^{2}$ law. Therefore, alternative theories are any way important to study.

Since the observed universe is almost homogeneous and isotropic, space-time is usually described by a Friedman-Lemaitre-Robertson-Walker (FLRW) cosmology. But it is also believed that in the early universe the FLRW model does not give a correct matter description. The anomalies found in the cosmic microwave background $(\mathrm{CMB})$ and the large structure observations stimulated a growing interest in anisotropic cosmological model of the universe. Observations by the Differential Macrowave Radiometers (DMR) on NASA's Cosmic Background Explorer Spacecraft registered anisotropy in various angle scales. It is conjectured, that these anisotropies hide in their hearts the entire history of the cosmic evolution down to recombination, and they are considered to be indicative of the universe geometry and the matter composing the universe. It is expected, that much more will be known about anisotropy of cosmic microwave's background after the investigations of the microwave's anisotropy probe. There is a general agreement among cosmologists that cosmic microwave's 
background anisotropy in the small angle scale holds the key to the formation of the discrete structure. The theoretical argument [1] and the modern experimental data support the existence of an anisotropic phase, which turns into an isotropic one.

In recent years, there has been considerable interest in scalar tensor theories of gravitation which are considered to be essential to describe the gravitational interaction near the planks scale, string theory, extended inflation and many higher order theories imply scalar field. Scalar-Tensor theories of gravitation provide the natural generalizations of general relativity and also provide a convenient set of representations for the observational limits on possible deviations from general relativity. There are two categories of gravitational theories involving a classical scalar field $\phi$. In first category the scalar field $\phi$ has the dimension of the inverse of the gravitational constant $\mathrm{G}$ among which the Brans-Decke theory [2] is of considerable importance and the role of the scalar field is confined to its effect on gravitational field equations. Brans and Decke formulated a scalartensor theory of gravitation which introduces an additional scalar field $\phi$ besides the metric tensor $g_{i j}$ and a dimensionless coupling constant $\omega$. This theory goes to general relativity for large values of the coupling constant $\omega>500$. In the second category of theories involve a dimensionless scalar field. Sáez and Ballester $[3]$ developed a scalar-tensor theory in which the metric is coupled with a dimensionless scalar field in a simple manner. This coupling gives a satisfactory description of the weak fields. In spite of the dimensionless character of the scalar field, an anti-gravity regime appears. This theory suggests a possible way to solve the missing-matter problem in non-flat FRW cosmologies. The Scalar-Tensor theories of gravitation play an important role to remove the graceful exit problem in the inflation era [4. In earlier literature, cosmological models within the framework of Sáez-Ballester scalar-tensor theory of gravitation, have been studied by Singh and Agrawal [5, 6, Ram and Tiwari [7, Singh and Ram [8. Mohanty and Sahu [9, 10] have studied Bianchi type- $\mathrm{VI}_{0}$ and Bianchi type-I models in Saez-Ballester theory. In recent years, Tripathi et al. 11, Reddy et al. 12, 13, Reddy and Naidu [14, Rao et al. 15, 16, 17, Adhav et al. [18, Katore et al. [19, Sahu [20, Singh [21] and Pradhan and Singh [22] have obtained the solutions in Sáez-Ballester scalar-tensor theory of gravitation in different context. Recently, Naidu et al. 23] and Reddy et al. 24] have studied LRS Bianchi type-II and five dimensional dark energy models in Sáez and Ballester scalar tensor theory of gravitation respectively.

Recently, Kumar and Singh [25] obtained exact Bianchi type-I cosmological models in Sáez and Ballester Scalar-Tensor theory of gravitation by assuming the constant deceleration parameter. In literature it is common to use a constant deceleration parameter, as it duly gives a power law for metric function or corresponding quantity. Several cosmological observations indicate that the observable universe is undergoing a phase of accelerated expansion (Riess et al. 26]; Perlmutter et al. [27; Bennet et al. 28]; Tegmark et al. [29]; Allen et al. [30]). Recent observations of SNe Ia of high confidence level (Tonry et al. [31; Riess et al. [32]; Clocchiatti et al. 333]) have further confirmed this. Also, the transition redshift from deceleration expansion to accelerated expansion is about 0.5. Now for a Universe which was decelerating in past and accelerating at the present time, the DP must show signature flipping (see the Refs. Padmanabhan and Roychowdhury [34, Amendola [35, Riess et al. [36]). So, in general, the DP is not a constant but time variable.

Socorro et al. 37 and Jamil et al. 38 have studied on anisotropic Bianchi type-I cosmology in Sáez-Ballester scalar-tensor theory of gravity. Recently, Pradhan et al. [39] have studied some exact Bianchi type-I cosmological models in scalar-tensor theory of gravitation with time dependent deceleration parameter. Motivated by the discussions, in this paper, we have obtained a new class of exact solutions of field equations given by Sáez and Ballester [3] in Bianchi type-I space-time by considering a time dependent deceleration parameter. The out line of the paper is as follows: In Section 2, the metric with a Lagrangian and the field equations are described. Section 3 deals with the solutions of the field equations. Subsections 3.1, 3.2 and 3.3 describe the three different models by considering the three different values of scale factors which yield the time dependent deceleration parameters and physical and geometric behaviour of the model are also discussed. Finally, conclusions are summarized in the last Section 4.

\section{The Metric and Field Equations}

We consider spatially homogeneous and anisotropic Bianchi type- $I$ space-time given by

$$
d s^{2}=-d t^{2}+A^{2} d x^{2}+B^{2} d y^{2}+C^{2} d z^{2},
$$


where the metric potentials $A, B$ and $C$ are functions of cosmic time $t$ alone. This ensures that the model is spatially homogeneous.

We, consider the simple case of a homogeneous but anisotropic Bianchi type-I model with matter term with a scalar field $\phi$. Our model is based on a non-standard scalar-tensor theory, defined in Sáez and Ballester [3] with a dimensionless scalar field $\phi$ and tensor field $g_{i j}$. This alternative theory of gravitation is combined scalar and tensor fields in which the metric is coupled with a dimensionless scalar field. We assume the Lagrangian

$$
L=R-\omega \phi^{k} \phi_{, i} \phi^{, i},
$$

$R$ being the scalar curvature, $\phi$ a dimensionless scalar field, $\omega$ and $k$ arbitrary dimensionless constants and $\phi^{, i}$ the contraction $\phi_{, \alpha} g^{\alpha i}$. Here the partial derivatives are denoted by a comma and the covariant derivatives by a semicolon in the usual way.

From the above Lagrangian we can establish the action

$$
I=\int_{\sum}\left(L+\chi L_{m}\right)(-g)^{\frac{1}{2}} d x^{1} d x^{2} d x^{3} d x^{4},
$$

where $L_{m}$ is the matter Lagrangian, $g$ is the determinant of the matrix $g_{i j}, x^{i}$ are the coordinates, $\sum$ is an arbitrary region of integration and $\chi=8 \pi$ (we use geometrized units). When $k=0$, our model is just the Einstein gravity with a massless minimally coupled scalar field coupled to gravity. Different aspects of this model have been investigated in the literatures (already described in Sect. 1). By considering arbitrary independent variations of the metric and the scalar field vanishing at the boundary of $\sum$, the variation principle

$$
\delta I=0,
$$

leads to a generalized Einstein equation

$$
\begin{gathered}
G_{i j}-\omega \phi^{k}\left(\phi_{, i} \phi_{, j}-\frac{1}{2} g_{i j} \phi_{, l} \phi^{l}\right)=-8 \pi T_{i j}, \\
2 \phi^{k} \phi_{; i}^{, i}+k \phi^{k-1} \phi_{, l} \phi^{, l}=0
\end{gathered}
$$

where $G_{i j}=R_{i j}-\frac{1}{2} R g_{i j}$ is the Einstein tensor; $T_{i j}$ is the stress-energy tensor of the matter Lagrangian $L_{m}$.

Since the action $I$ is a scalar, it can be easily proved that the equation of motion

$$
T_{; i}^{i j}=0,
$$

are consequences of the field equations.

In Brans-Dicke gravity, the modification was introduced due to the lack of compatibility of Einstein's theory with Mach's principle. But in Sáez and Ballester [3] a scalar-tensor theory of gravity was introduced in which metric is coupled to scalar field. Here the strength of the coupling between gravity and the field was governed by a parameter $\omega$. With this modification, they were able to solve a 'missing-mass problem'. Several aspects of Sáez \& Ballester scalar-tensor theory of gravitation in relation to Bianchi cosmological models have been explored in literature.

The energy-momentum tensor $T_{i j}$ for a perfect fluid has the form

$$
T_{i j}=(p+\rho) u_{i} u_{j}-p g_{i j},
$$

where $p$ is the thermodynamical pressure, $\rho$ the energy density, $u_{i}$ the four-velocity of the fluid satisfying

$$
g_{i j} u^{i} u^{j}=1 \text {. }
$$

In co-moving system of coordinates, we have $u_{i}=(0,0,0,1)$. For the energy momentum tensor (7) and Bianchi type- $I$ space-time (1), Einstein's modified field equations (5) yield the following five independent equations

$$
\frac{\ddot{A}}{A}+\frac{\ddot{B}}{B}+\frac{\dot{A} \dot{B}}{A B}=-8 \pi p+\frac{1}{2} \omega \phi^{k} \dot{\phi}^{2},
$$




$$
\begin{aligned}
& \frac{\ddot{A}}{A}+\frac{\ddot{C}}{C}+\frac{\dot{A} \dot{C}}{A C}=-8 \pi p+\frac{1}{2} \omega \phi^{k} \dot{\phi}^{2}, \\
& \frac{\ddot{B}}{B}+\frac{\ddot{C}}{C}+\frac{\dot{B} \dot{C}}{B C}=-8 \pi p+\frac{1}{2} \omega \phi^{k} \dot{\phi}^{2}, \\
& \frac{\dot{A} \dot{B}}{A B}+\frac{\dot{A} \dot{C}}{A C}+\frac{\dot{B} \dot{C}}{B C}=8 \pi \rho-\frac{1}{2} \omega \phi^{k} \dot{\phi}^{2}, \\
& \ddot{\phi}+\dot{\phi}\left(\frac{\dot{A}}{A}+\frac{\dot{B}}{B}+\frac{\dot{C}}{C}\right)+\frac{m \dot{\phi}^{2}}{2 \phi}=0,
\end{aligned}
$$

where an over dot denotes derivative with respect to cosmic time $t$. The law of energy-conservation equation $\left(T_{; j}^{i j}=0\right)$ gives

$$
\dot{\rho}+(\rho+p)\left(\frac{\dot{A}}{A}+\frac{\dot{B}}{B}+\frac{\dot{C}}{C}\right)=0 .
$$

It is worth noting here that our approach suffers from a lack of Lagrangian approach. There is no known way to present a consistent Lagrangian model satisfying the necessary conditions discussed in this paper.

The spatial volume for the model (11) is given by

$$
V^{3}=A B C .
$$

We define $a=(A B C)^{\frac{1}{3}}$ as the average scale factor so that the Hubble's parameter is anisotropic and may be defined as

$$
H=\frac{\dot{a}}{a}=\frac{1}{3}\left(\frac{\dot{A}}{A}+\frac{\dot{B}}{B}+\frac{\dot{C}}{C}\right)
$$

We also have

$$
H=\frac{1}{3}\left(H_{x}+H_{y}+H_{z}\right)
$$

where $H_{x}=\frac{\dot{A}}{A}, H_{y}=\frac{\dot{B}}{B}$ and $H_{z}=\frac{\dot{C}}{C}$.

The deceleration parameter $q$, the scalar expansion $\theta$, shear scalar $\sigma^{2}$ and the average anisotropy parameter $A_{m}$ are defined by

$$
\begin{gathered}
q=-\frac{a \ddot{a}}{\dot{a}^{2}}=-\frac{\ddot{a}}{a H^{2}}, \\
\theta=\frac{\dot{A}}{A}+\frac{\dot{B}}{B}+\frac{\dot{C}}{C}, \\
\sigma^{2}=\frac{1}{2}\left(\sum_{i=1}^{3} H_{i}^{2}-\frac{1}{3} \theta^{2}\right), \\
A_{m}=\frac{1}{3} \sum_{i=1}^{3}\left(\frac{\triangle H_{i}}{H}\right)^{2},
\end{gathered}
$$

where $\triangle H_{i}=H_{i}-H(i=x, y, z)$.

\section{Solution of the Field Equations}

The field equations (9)-(13) are five equations necessitating six unknowns $A, B, C, p, \rho$ and $\phi$. One additional constraint relating these parameters is required to obtain explicit solutions of the system. We consider the deceleration parameter as time dependent to get the deterministic solution.

We have revisited the solutions of Kumar and Singh [25. Now subtracting (9) from (10), (9) from (11), (10) from (11) and taking second integral of each expression, we obtain the following three relations, respectively

$$
\frac{A}{B}=d_{1} \exp \left(x_{1} \int a^{-3} d t\right),
$$




$$
\begin{aligned}
& \frac{A}{C}=d_{2} \exp \left(x_{2} \int a^{-3} d t\right), \\
& \frac{B}{C}=d_{3} \exp \left(x_{3} \int a^{-3} d t\right),
\end{aligned}
$$

where $d_{1}, d_{2}, d_{3}, x_{1}, x_{2}$ and $x_{3}$ are integrating constants.

From above Eqs. (22)-(24), the metric functions $A(t), B(t)$ and $C(t)$ are explicitly obtained as

$$
\begin{aligned}
& A(t)=a_{1} a \exp \left(b_{1} \int a^{-3} d t\right), \\
& B(t)=a_{2} a \exp \left(b_{2} \int a^{-3} d t\right), \\
& C(t)=a_{3} a \exp \left(b_{3} \int a^{-3} d t\right),
\end{aligned}
$$

where

$$
\begin{gathered}
a_{1}=\left(d_{1} d_{2}\right)^{\frac{1}{3}}, \quad a_{2}=\left(\frac{d_{3}}{d_{1}}\right)^{\frac{1}{3}}, a_{3}=\left(d_{2} d_{3}\right)^{-\frac{1}{3}}, \\
b_{1}=\frac{1}{3}\left(x_{1}+x_{2}\right), \quad b_{2}=\frac{1}{3}\left(x_{3}-x_{1}\right), \quad b_{3}=-\frac{1}{3}\left(x_{2}+x_{3}\right) .
\end{gathered}
$$

It is worth mention here that these constants also satisfy the following two conditions

$$
a_{1} a_{2} a_{3}=1, \quad b_{1}+b_{2}+b_{3}=0 .
$$

The second integral of (13) leads to

$$
\phi(t)=\left[\frac{h(k+2)}{2} \int a^{-3} d t\right]^{\frac{2}{(m+2}},
$$

where $h$ is a constant due to first integral while the constant of second integral is taken as zero for simplicity without any loss of generality.

\subsection{Case 1: when $a(t)=\sqrt{t^{n} e^{t}}$}

Following Saha et al. [40] and Pradhan and Amirhashchi [41, we take following ansatz for the scale factor, where increase in term of time evolution is

$$
a(t)=\sqrt{t^{n} e^{t}},
$$

where $n$ is a positive constant. This ansatz generalized the one proposed by Amirhashchi et al. [42]. The motivation to choose such scale factor (31) yields a time-dependent DP. Recently Yadav [43] has studied such type of scale factor in Bianchi- $\mathrm{V}$ string cosmological model at late time acceleration.

Substituting Eq. (31) into Eq. (18), we find

$$
q=\frac{2 n}{(n+t)^{2}}-1 .
$$

From Eq. (32), we observe that $q>0$ for $t<\sqrt{2 n}-n$ and $q<0$ for $t>\sqrt{2 n}-n$. It is observed that for $0<n<2$, our model is evolving from deceleration phase to acceleration phase. Also, recent observations of $\mathrm{SNe}$ Ia, expose that the present universe is accelerating and the value of DP lies to some place in the range $-1<q<0$. It follows that in our derived model, one can choose the value of DP consistent with the observation. Figure 1 graphs the deceleration parameter $(q)$ versus time which gives the behaviour of $q$ from decelerating to accelerating phase for different values of $n$ which is consistent with recent observations of Type Ia supernovae (Riess et al. [26]; Perlmutter et al. 227; Tonry et al. [31]; Riess et al. [32]; Clocchiatti et al. [33]). 


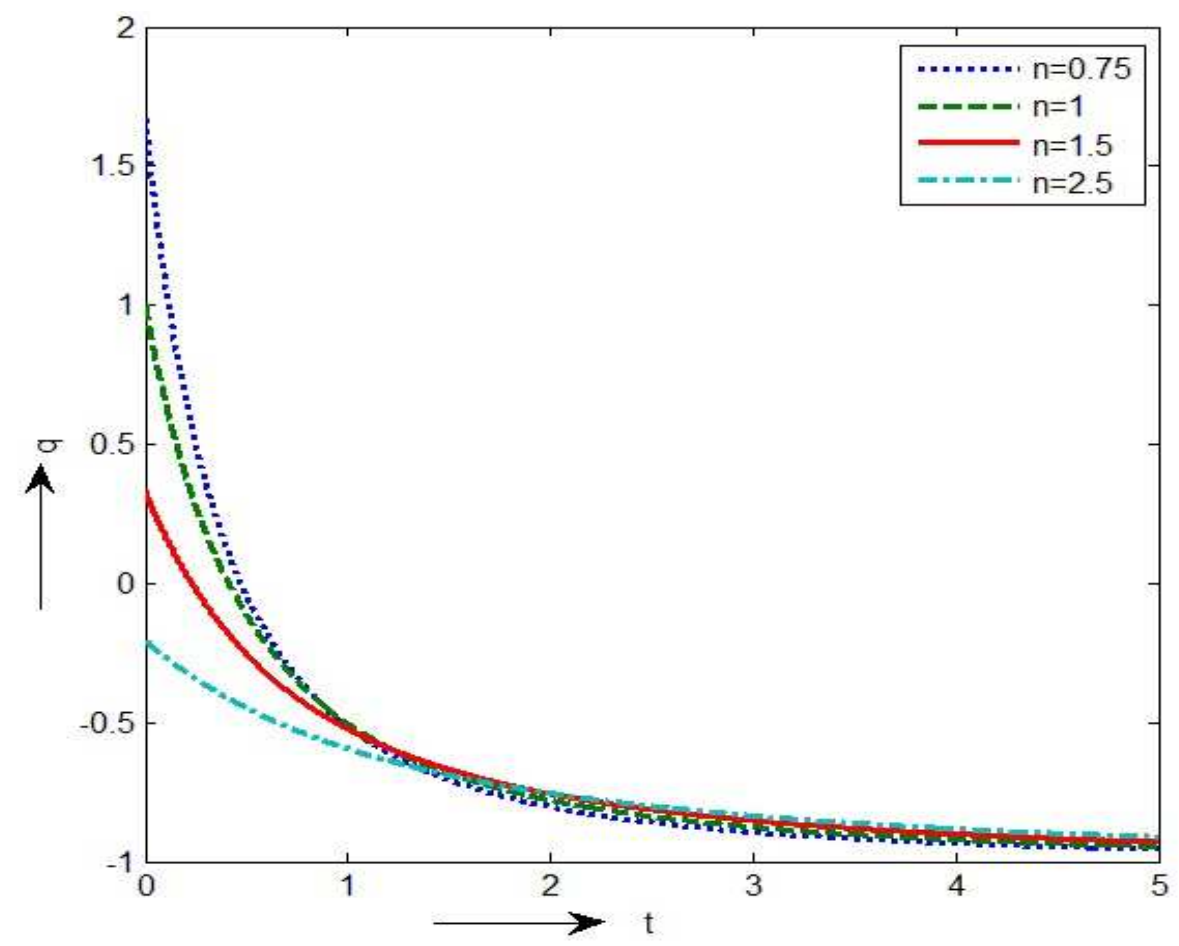

Figure 1: The plot of deceleration parameter $q$ versus $t$

Using Eq. (31) in (25)-(27), we get the following expressions for metric coefficients

$$
\begin{aligned}
& A=a_{1}\left(\sqrt{t^{n} e^{t}}\right) \exp \left[b_{1} \int\left(t^{n} e^{t}\right)^{-\frac{3}{2}} d t\right], \\
& B=a_{2}\left(\sqrt{t^{n} e^{t}}\right) \exp \left[b_{2} \int\left(t^{n} e^{t}\right)^{-\frac{3}{2}} d t\right], \\
& C=a_{3}\left(\sqrt{t^{n} e^{t}}\right) \exp \left[b_{3} \int\left(t^{n} e^{t}\right)^{-\frac{3}{2}} d t\right] .
\end{aligned}
$$

Again substituting (31) in (30), the scalar field is obtained as

$$
\phi=\left[\frac{h(k+2)}{2} \int\left(t^{n} e^{t}\right)^{-\frac{3}{2}} d t\right]^{\frac{2}{(k+2)}},
$$

Eq. (36) implies that

$$
\phi^{k} \dot{\phi}^{2}=h^{2}\left(t^{n} e^{t}\right)^{-3} .
$$

Substituting (33)-(37) in (11) and (12), the expressions for thermodynamical pressure $(p)$ and energy density $(\rho)$ for the model are obtained as

$$
\begin{gathered}
p=\frac{n}{t^{2}}-\frac{3}{4}\left(\frac{n}{t}+1\right)^{2}+\left(\frac{1}{2} \omega h^{2}-\beta_{3}\right)\left(t^{n} e^{t}\right)^{-3}, \\
\rho=\frac{3}{4}\left(\frac{n}{t}+1\right)^{2}+\left(\frac{1}{2} \omega h^{2}+\beta_{2}\right)\left(t^{n} e^{t}\right)^{-3},
\end{gathered}
$$

where

$$
\begin{gathered}
\beta_{1}=b_{1}^{2}+b_{2}^{2}+b_{3}^{2}, \\
\beta_{2}=b_{1} b_{2}+b_{2} b_{3}+b_{3} b_{1}, \\
\beta_{3}=b_{2}^{2}+b_{3}^{2}+b_{2} b_{3} .
\end{gathered}
$$




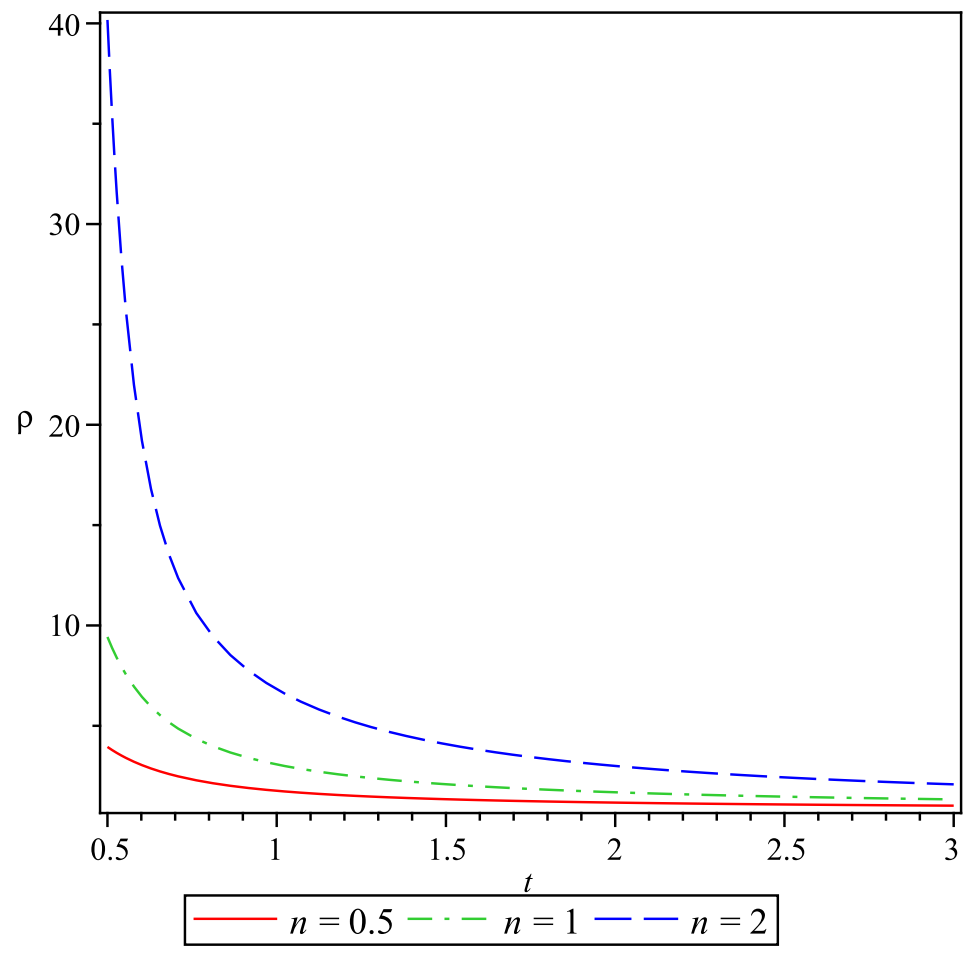

Figure 2: The plot of energy density $\rho$ versus $t$. Here $\omega=1, h=\beta_{2}=1$

In view of (29), it is observed that the solutions given by (33)-(40) satisfy the energy conservation equation (14) identically and hence represent exact solutions of the Einstein's modified field equations (9)-(13). From Eq. (39), it is observed that $\rho$ is a positive decreasing function of time and it approaches to zero as $t \rightarrow \infty$. This behaviour is clearly depicted in Figure 2 as a representative case with appropriate choice of constants of integrations and other physical parameters using reasonably well known situations.

The rate of expansion $H_{i}$ in the direction of $x, y$, and $z$ read as

$$
\begin{aligned}
& H_{x}=\frac{1}{2}\left(\frac{n}{t}+1\right)+b_{1}\left(t^{n} e^{t}\right)^{-\frac{3}{2}}, \\
& H_{y}=\frac{1}{2}\left(\frac{n}{t}+1\right)+b_{2}\left(t^{n} e^{t}\right)^{-\frac{3}{2}}, \\
& H_{z}=\frac{1}{2}\left(\frac{n}{t}+1\right)+b_{3}\left(t^{n} e^{t}\right)^{-\frac{3}{2}} .
\end{aligned}
$$

The Hubble parameter, expansion scalar and shear of the model are, respectively given by

$$
\begin{aligned}
H & =\frac{1}{2}\left(\frac{n}{t}+1\right), \\
\theta & =\frac{3}{2}\left(\frac{n}{t}+1\right), \\
\sigma^{2} & =\frac{1}{2} \beta_{1}\left(t^{n} e^{t}\right)^{-3},
\end{aligned}
$$

where $\beta_{1}$ is given in Eq. (40).

The spatial volume $(V)$ and anisotropy parameter $\left(A_{m}\right)$ are found to be

$$
V=\left(t^{n} e^{t}\right)^{\frac{3}{2}}
$$




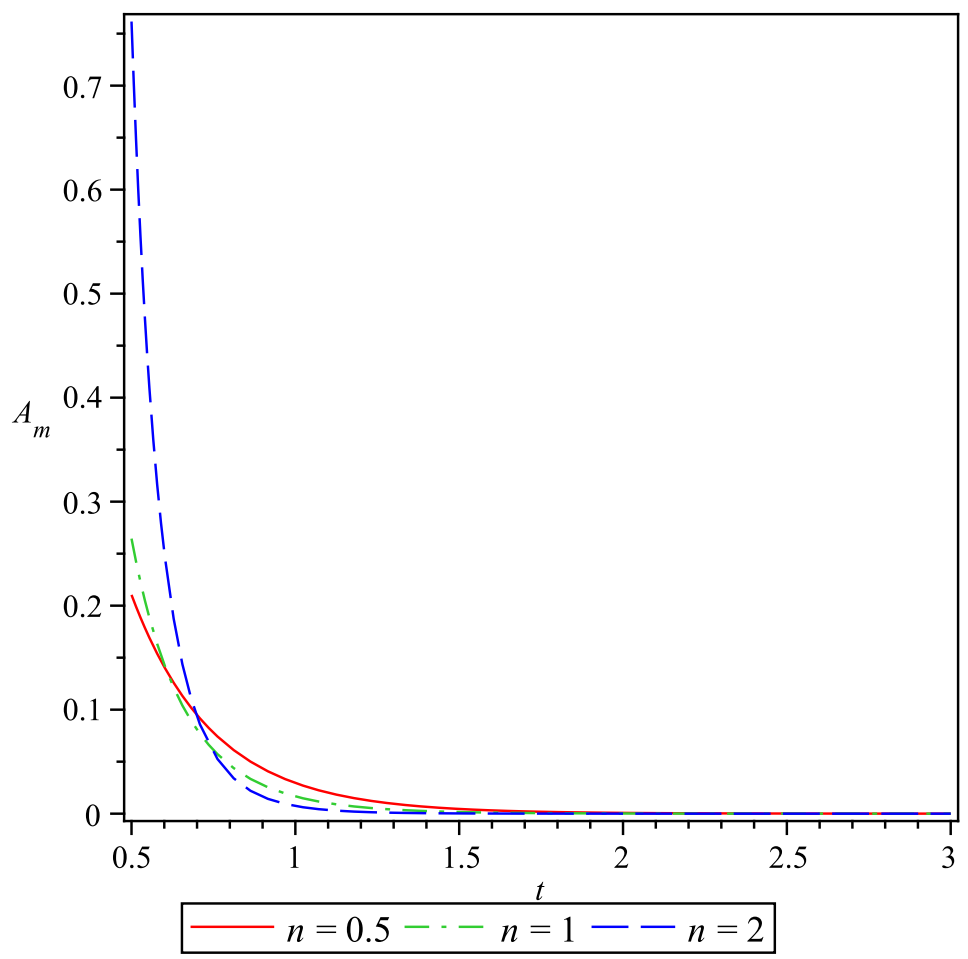

Figure 3: The plot of anisotropic parameter $A_{m}$ versus $t$. Here $\beta_{1}=1$

$$
A_{m}=\frac{4}{3} \beta_{1}\left(t^{n} e^{t}\right)^{-3}\left(\frac{n}{t}+1\right)^{-2} .
$$

From Eqs. (47) and (45) we observe that the spatial volume is zero at $t=0$ and the expansion scalar is infinite, which show that the universe starts evolving with zero volume at $t=0$ which is big bang scenario. From Eqs. (33)-(35), we observe that the spatial scale factors are zero at the initial epoch $t=0$ and hence the model has a point type singularity [46]). We observe that proper volume increases with time.

The dynamics of the mean anisotropic parameter depends on the constant $\beta_{1}=b_{1}^{2}+b_{2}^{2}+b_{3}^{2}$. From Eq. (48), we observe that at late time when $t \rightarrow \infty, A_{m} \rightarrow 0$. Thus, our model has transition from initial anisotropy to isotropy at present epoch which is in good harmony with current observations. Figure 3 depicts the variation of anisotropic parameter $\left(A_{m}\right)$ versus cosmic time $t$. From the figure, we observe that $A_{m}$ decreases with time and tends to zero as $t \rightarrow \infty$. Thus, the observed isotropy of the universe can be achieved in our model at present epoch.

It is important to note here that $\lim _{t \rightarrow 0}\left(\frac{\rho}{\theta^{2}}\right)$ spread out to be constant. Therefore the model of the universe goes up homogeneity and matter is dynamically negligible near the origin. This is in good agreement with the result already given by Collins [44.

\subsection{Case 2: when $a(t)=\sqrt{t e^{t}}$}

Following Amirhashchi et al. [42, we consider the following ansatz for the scale factor, where the increase in terms of time evolution is

$$
a(t)=\sqrt{t e^{t}} .
$$

By the above choice of scale factor yields a time dependent deceleration parameter. Using (49) into (18), we find

$$
q=\frac{2}{(1+t)^{2}}-1 .
$$


Using Eq. (49) in (33)-(35), we get the following expressions for metric coefficients

$$
\begin{aligned}
& A=a_{1}\left(\sqrt{t e^{t}}\right) \exp \left[b_{1} \int\left(t e^{t}\right)^{-\frac{3}{2}} d t\right], \\
& B=a_{2}\left(\sqrt{t e^{t}}\right) \exp \left[b_{2} \int\left(t e^{t}\right)^{-\frac{3}{2}} d t\right], \\
& C=a_{3}\left(\sqrt{t e^{t}}\right) \exp \left[b_{3} \int\left(t e^{t}\right)^{-\frac{3}{2}} d t\right],
\end{aligned}
$$

where the constants are already defined in previous section.

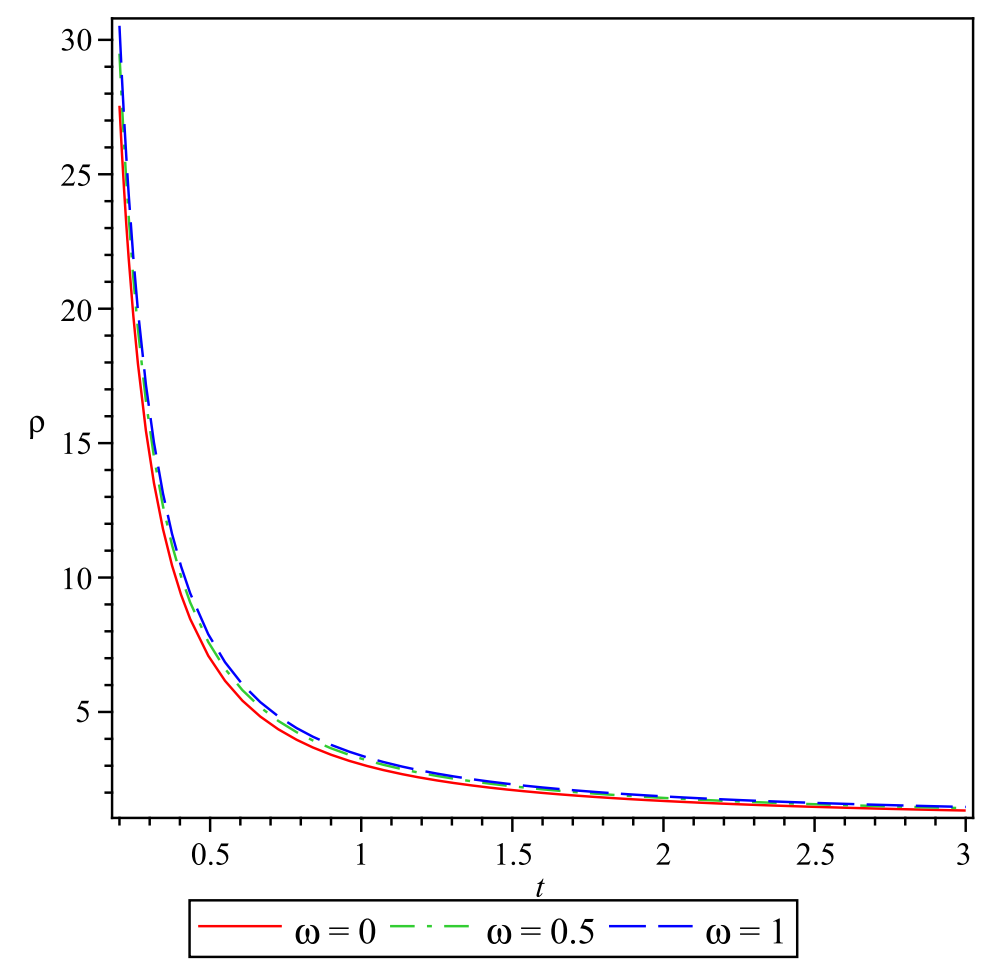

Figure 4: The plot of energy density $\rho$ versus $t$. Here $h=\beta_{2}=1$

Again substituting (49) in (30), the scalar field is obtained as

$$
\phi=\left[\frac{h(k+2)}{2} \int\left(t e^{t}\right)^{-\frac{3}{2}} d t\right]^{\frac{2}{(k+2)}},
$$

Eq. (54) implies that

$$
\phi^{k} \dot{\phi}^{2}=h^{2}\left(t e^{t}\right)^{-3} .
$$

Substituting (51)-(55) in (11) and (12), the expressions for thermodynamical pressure $(p)$ and energy density $(\rho)$ for the model are obtained as

$$
\begin{gathered}
p=\frac{1}{t^{2}}-\frac{3}{4}\left(\frac{1}{t}+1\right)^{2}+\left(\frac{1}{2} \omega h^{2}-\beta_{3}\right)\left(t e^{t}\right)^{-3}, \\
\rho=\frac{3}{4}\left(\frac{1}{t}+1\right)^{2}+\left(\frac{1}{2} \omega h^{2}+\beta_{2}\right)\left(t e^{t}\right)^{-3},
\end{gathered}
$$

where $\beta_{2}$ and $\beta_{3}$ are already given in previous section. 


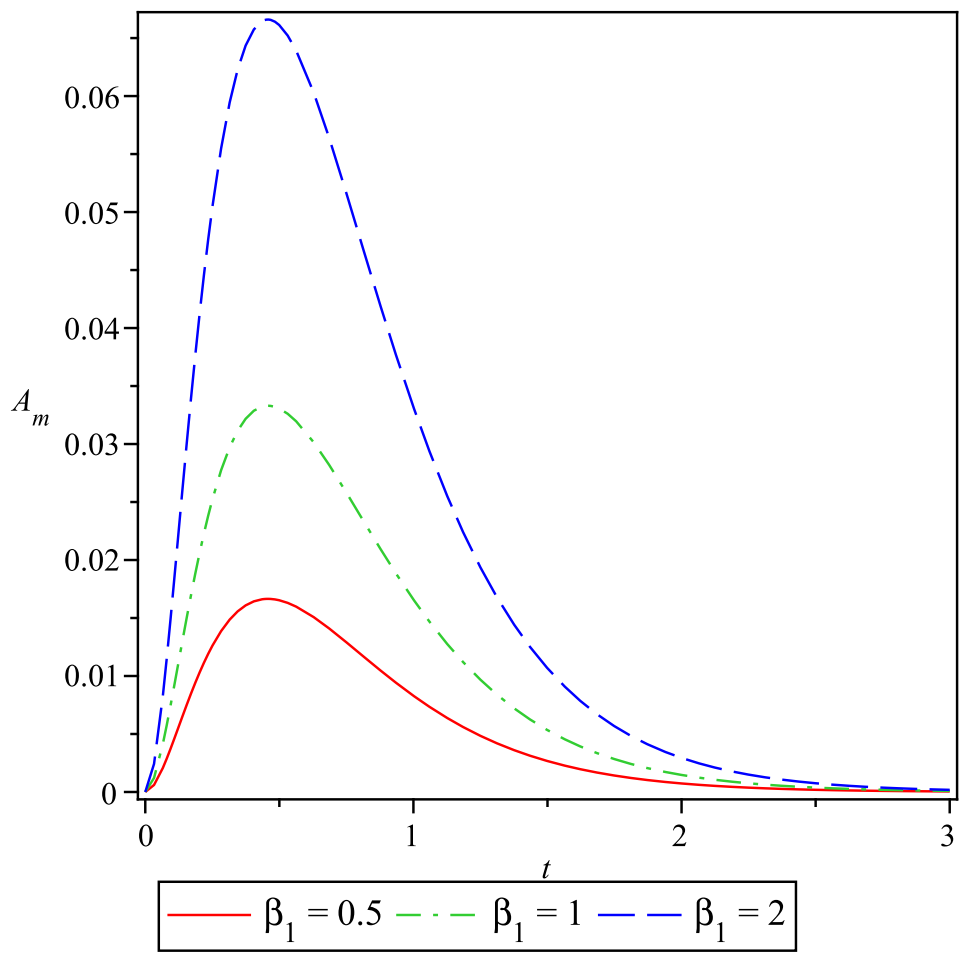

Figure 5: The plot of anisotropic parameter $A_{m}$ versus $t$

In view of (29), it is observed that the solutions given by (51)-(57) satisfy the energy conservation equation (14) identically and hence represent exact solutions of the Einstein's modified field equations (9)-(13). From Eq. (57), it is observed that $\rho$ is a positive decreasing function of time and it approaches to zero as $t \rightarrow \infty$. Figure 4 shows this behaviour of $\rho$.

The rate of expansion $H_{i}$ in the direction of $x, y$, and $z$ read as

$$
\begin{aligned}
& H_{x}=\frac{1}{2}\left(\frac{1}{t}+1\right)+b_{1}\left(t e^{t}\right)^{-\frac{3}{2}}, \\
& H_{y}=\frac{1}{2}\left(\frac{1}{t}+1\right)+b_{2}\left(t e^{t}\right)^{-\frac{3}{2}}, \\
& H_{z}=\frac{1}{2}\left(\frac{1}{t}+1\right)+b_{3}\left(t e^{t}\right)^{-\frac{3}{2}} .
\end{aligned}
$$

The Hubble parameter, expansion scalar and shear of the model are, respectively given by

$$
\begin{aligned}
H & =\frac{1}{2}\left(\frac{1}{t}+1\right), \\
\theta & =\frac{3}{2}\left(\frac{1}{t}+1\right), \\
\sigma^{2} & =\frac{1}{2} \beta_{1}\left(t e^{t}\right)^{-3},
\end{aligned}
$$

The spatial volume $(V)$ and anisotropy parameter $\left(A_{m}\right)$ are found to be

$$
\begin{gathered}
V=\left(t e^{t}\right)^{\frac{3}{2}} \\
A_{m}=\frac{4}{3} \beta_{1}\left(t e^{t}\right)^{-3}\left(\frac{1}{t}+1\right)^{-2} .
\end{gathered}
$$


where $\beta_{1}$ is already defined in previous section.

This model has the same properties as described in Case1. This is a particular case of previous one.

\subsection{Case 3: when $a(t)=-\frac{1}{t}+t^{2}$}

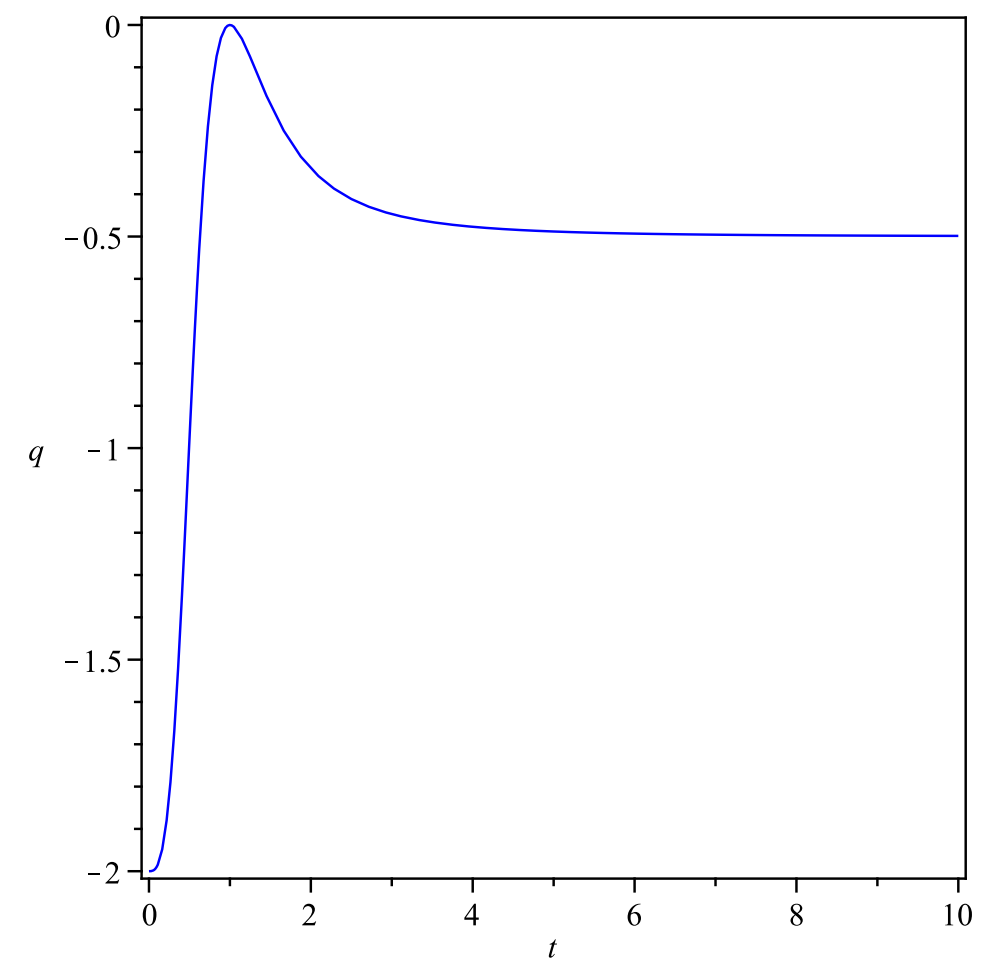

Figure 6: The plot of deceleration parameter $q$ versus $t$

In this case, we consider the following ansatz for the scale factor, where the increase in terms of time evolution is

$$
a(t)=-\frac{1}{t}+t^{2},
$$

which yields a time dependent deceleration parameter. Using (66) into (18), we find

$$
q=-2\left(\frac{t^{3}-1}{2 t^{3}+1}\right)^{2} .
$$

From Eq. (67), we observe that $q=-2$ at $t=0$ and $q=-0.5$ as $t \rightarrow \infty$. Figure 6 depicts the variation of deceleration parameter versus cosmic time $t$. From figure we observe that $q=-2$ at $t=0$ and increases very rapidly to reach its maximum near zero and then decreases with time and ultimately it becomes constant $(-0.5)$ as $t \rightarrow \infty$. The values of the deceleration parameter separate decelerating $(q>0)$ from accelerating $(q<0)$ periods in the evolution of the universe. Determination of the deceleration parameter from the count magnitude relation for galaxies is a difficult task due to the evolutionary effects. The present value $q_{0}$ of the deceleration parameter obtained from observations are $-1.27 \leq q_{0} \leq 2$ (Schuecker et al. [45]). Studies of galaxy counts from redshift surveys provide a value of $q_{0}=0.1$, with an upper limit of $q_{0}<0.75$ [45]. Recent observations show that the deceleration parameter of the universe is in the range $-1 \leq q \leq 0$ i.e $q_{0} \approx-0.77$ [46]. Thus we see that the value of $q$ at present epoch in our derived model is very near to the value obtained by recent observation.

Using Eq. (66) in (33)-(35), we get the following expressions for metric coefficients

$$
A=a_{1}\left(-\frac{1}{t}+t^{2}\right) \exp \left[b_{1}\left(-\frac{1}{t}+t^{2}\right) d t\right]
$$




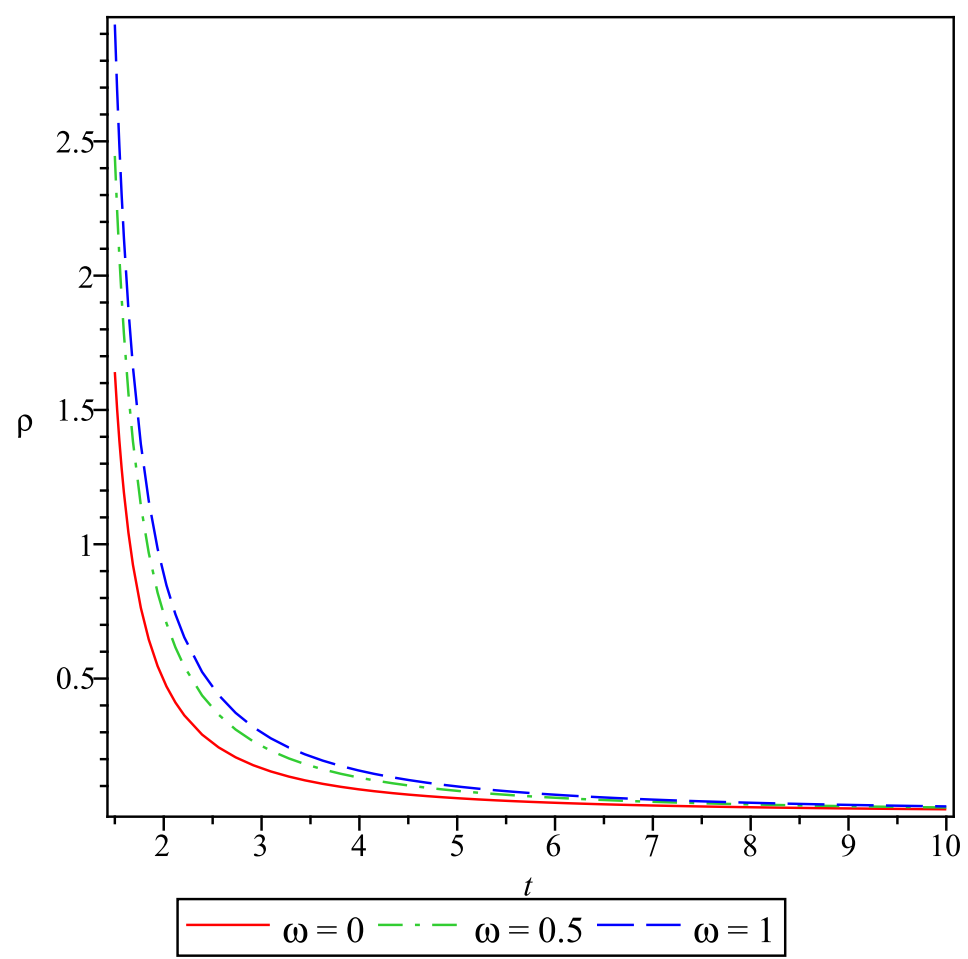

Figure 7: The plot of energy density $\rho$ versus $t$. Here $h=\beta_{2}=1$

$$
\begin{aligned}
& B=a_{2}\left(-\frac{1}{t}+t^{2}\right) \exp \left[b_{2}\left(-\frac{1}{t}+t^{2}\right) d t\right], \\
& C=a_{3}\left(-\frac{1}{t}+t^{2}\right) \exp \left[b_{3}\left(-\frac{1}{t}+t^{2}\right) d t\right],
\end{aligned}
$$

where the constants are already defined in previous section 3.1 .

Again substituting (66) in (30), the scalar field is obtained as

$$
\phi=\left[\frac{h(k+2)}{2} \int\left(-\frac{1}{t}+t^{2}\right)^{-3} d t\right]^{\frac{2}{(k+2)}},
$$

Eq. (71) implies that

$$
\phi^{k} \dot{\phi}^{2}=h^{2}\left(-\frac{1}{t}+t^{2}\right)^{-6} .
$$

Substituting (68)-(72) in (11) and (12), the expressions for isotropic pressure $(p)$ and energy density $(\rho)$ for the model are obtained as

$$
\begin{aligned}
& p=\left(\frac{1}{2} \omega h^{2}-\beta_{3}\right)\left(-\frac{1}{t}+t^{2}\right)^{-6}-\frac{3\left(2 t^{2}+1\right)^{2}}{t^{2}\left(t^{3}-1\right)^{2}}, \\
& \rho=\left(\frac{1}{2} \omega h^{2}-\beta_{2}\right)\left(-\frac{1}{t}+t^{2}\right)^{-6}+\frac{3\left(2 t^{2}+1\right)^{2}}{t^{2}\left(t^{3}-1\right)^{2}},
\end{aligned}
$$

where $\beta_{2}$ and $\beta_{3}$ are already given in section 3.1.

In view of (29), it is observed that the solutions given by (68)-(74) satisfy the energy conservation equation (14) identically and hence represent exact solutions of the Einstein's modified field equations (91)-(13). From Eq. (74), it is observed that $\rho$ is a positive decreasing function of time and it approaches to zero as $t \rightarrow \infty$. Figure 


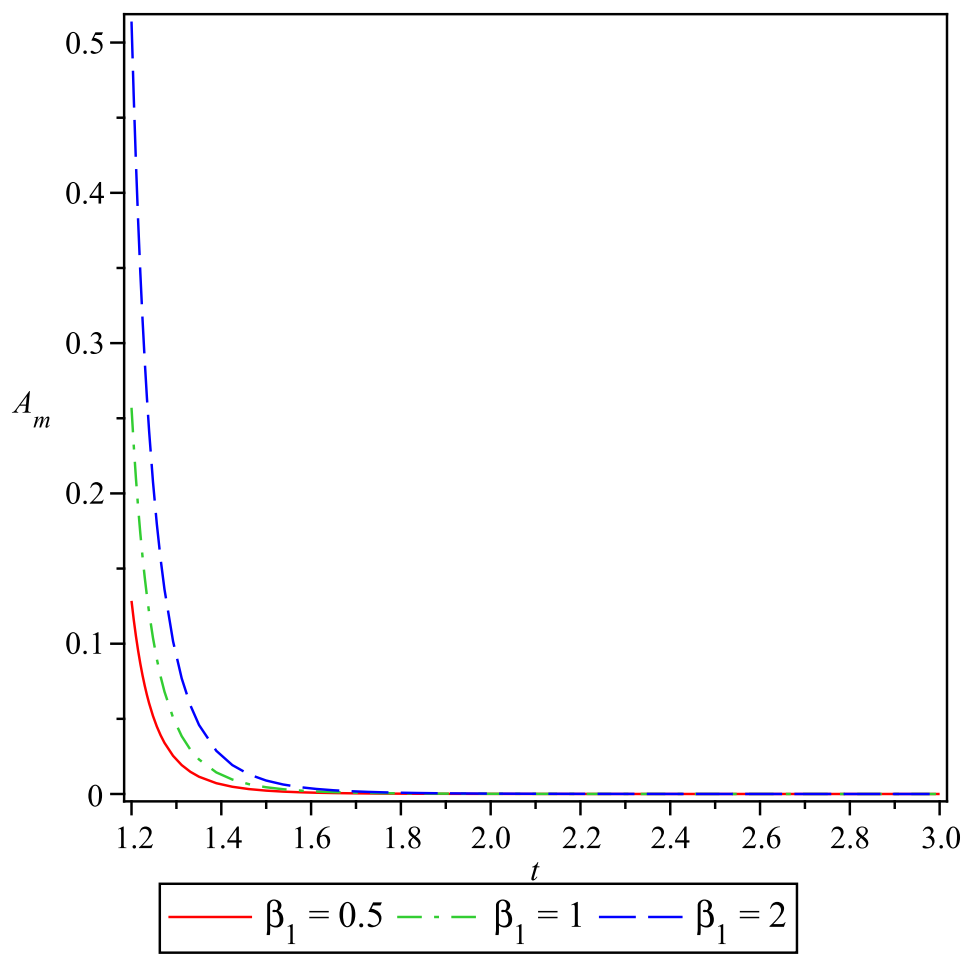

Figure 8: The plot of anisotropic parameter $A_{m}$ versus $t$

7 depicts the variation of energy density versus cosmic time $t$. We observe from the figure that $\rho$ is a positive decreasing function of time and it approaches to zero at late time.

The rate of expansion $H_{i}$ in the direction of $x, y$, and $z$ read as

$$
\begin{aligned}
& H_{x}=\frac{\left(2 t^{3}+1\right)}{\left(t^{3}-1\right) t}+b_{1}\left(-\frac{1}{t}+t^{2}\right)^{-3}, \\
& H_{y}=\frac{\left(2 t^{3}+1\right)}{\left(t^{3}-1\right) t}+b_{2}\left(-\frac{1}{t}+t^{2}\right)^{-3}, \\
& H_{z}=\frac{\left(2 t^{3}+1\right)}{\left(t^{3}-1\right) t}+b_{3}\left(-\frac{1}{t}+t^{2}\right)^{-3} .
\end{aligned}
$$

The Hubble parameter, expansion scalar and shear of the model are, respectively given by

$$
\begin{gathered}
H=\frac{\left(2 t^{3}+1\right)}{\left(t^{3}-1\right) t}, \\
\theta=\frac{3\left(2 t^{3}+1\right)}{\left(t^{3}-1\right) t}, \\
\sigma^{2}=\frac{1}{2} \beta_{1}\left(-\frac{1}{t}+t^{2}\right)^{-6}
\end{gathered}
$$

The spatial volume $(V)$ and anisotropy parameter $\left(A_{m}\right)$ are found to be

$$
\begin{gathered}
V=\left(-\frac{1}{t}+t^{2}\right)^{3} . \\
A_{m}=\frac{1}{3} \beta_{1} \frac{\left(t^{3}-1\right)^{2} t^{2}}{\left(2 t^{3}+1\right)^{2}}\left(-\frac{1}{t}+t^{2}\right)^{-6} .
\end{gathered}
$$


where $\beta_{1}$ is already defined in previous section.

It is observed that the spatial volume is zero at $t=1$ and the expansion scalar is infinite, which shows that the universe starts evolving with zero volume at initial epoch $t=1$ with initial rate of expansion. The scale factors also vanish at $t=1$ and hence the model has a Point type singularity 47] at the initial epoch. The pressure, energy density, Hubble's factors and shear scalar diverge at the initial singularity. Figure 8 is the plot of the anisotropic parameter $\left(A_{m}\right)$ versus cosmic time $t$. From the figure, we observe that $A_{m}$ decreases with time and tends to zero as $t \rightarrow \infty$ for all values of $\beta_{1}$. Thus, the observed isotropy of the universe can be achieved in our derived model at present epoch. The shear tensor also tends to zero in this model. Thus the model represents shearing, non-rotating and expanding model of the universe with a big bang start approaching to isotropy at late times.

\section{Concluding Remarks}

In this paper we have studied a spatially homogeneous and anisotropic Bianchi type-I space-time within the framework of the scalar-tensor theory of gravitation proposed by Sáez and Ballester 3 . The field equations have been solved exactly with suitable physical assumption. The solutions in all three cases satisfy the energy conservation Eq. (14) identically. Therefore, exact and physically viable Bianchi type-I models have been obtained. To find the deterministic solution, we have considered three types of scale factors which yield three different time dependent deceleration parameters.

In Case 1, we choosed the scale factor $a(t)=\sqrt{t^{n} e^{t}}$ which yields a time dependent deceleration parameter $q=\frac{2 n}{(n+t)^{2}}-1$. Since all the scale factors vanish at initial moment, hence the model has a big bang singularity at $t=0$. This is a Point type singularity. This case represents a model which generates a transition of the universe from early decelerated phase to the recent accelerating phase which is in good agreement with the recent observations (Caldwell et al. [48]) (see, Fig. 1) whereas in Pradhan et al. [39] only the evolution takes place in an accelerating phase. The model has also transition from initial anisotropy to isotropy at late time (i.e. present epoch) which is in good harmony with current observations (see, Fig. 3). For different choice of $n$, we can generate a class of cosmological models in Bianchi type-I space-time. It is observed that such models are also in good harmony with current observations [26, 27, 31-33].

In Case 2, we considered the scale factor $a(t)=\sqrt{t e^{t}}$ which yields a time dependent deceleration parameter $q=\frac{2}{(1+t)^{2}}-1$. This is a particular case of previous one. This model has the same properties as obtained in Case 1. It is worth mention here that the anisotropic parameter becomes negligible at $t \rightarrow \infty$ which, in turn, implies that the derived model isotropizes at present epoch.

In Case 3, we considered the scale factor $a(t)=-\frac{1}{t}+t^{2}$ which yields a time dependent deceleration parameter $q=-2\left(\frac{t^{3}-1}{2 t^{3}+1}\right)^{2}$. In this case we get an accelerating universe (see, Fig. 6) at present epoch. In this case, the anisotropic behaviour of the universe dies out on later times and the observed isotropy of the universe can be achieved in derived model at present epoch (see, Fig. 8). The model has a point type singularity at initial epoch $t=1$.

It is worth mentioned here that in all above three cases, their anisotropic solutions may enter to some isotropic inflationary era. All the above three models represent expanding, shearing and non-rotating universe, which approach to isotropy for large value of $t$. This is consistent with the behaviour of the present universe as already discussed in introduction. If we set $k=0$, then the solutions reduce to the solution in general relativity and the Sáez-Ballester scalar-tensor theory of gravitation tends to standard general theory of relativity in every respect. In literature we can get the solutions of the field equations of Sáez-Ballester scalar-tensor theory of gravitation by using a constant deceleration parameter. So the solutions presented in this paper are new and different from other author's solutions.

Finally, our solutions may be useful for better understanding of the evolution of the universe in Bianchi-I space-time within the framework of Sáez-Ballester scalar-tensor theory of gravitation. The solutions presented here can be one of the potential candidates to describe the observed universe. 


\section{Acknowledgement}

Author (A. Pradhan) would like to thank the Inter-University Centre for Astronomy and Astrophysics (IUCAA),

Pune, India for providing facility and support under associateship program where part of this work was carried out. The financial support (Project No. C.S.T./D-1536) in part by State Council of Science and Technology, Uttar Pradesh, India is gratefully acknowledged by A. Pradhan.

\section{References}

[1] Misner, C.W.: Astrophys. J. 151, 431 (1968)

[2] Brans, C.H., Dicke, R.H.: Phys. Rev. 124, 925 (1961)

[3] Sáez, D., Ballester, V.J.: Phys. Lett. 113, 467 (1986)

[4] Piemental, L.O.: Mod. Phys. Lett. A 12, 1865 (1997)

[5] Singh, T., Agrawal, A.K.: Astrophys. Space Sci. 182, 289 (1991)

[6] Singh, T., Agrawal, A.K.: Astrophys. Space Sci. 191, 61 (1992)

[7] Ram, S., Tiwari, S.K.: Astrophys. Space Sci. 259, 91 (1998)

[8] Singh, C.P., Ram, S.: Astrophys. Space Sci. 284, 1999 (2003)

[9] Mohanty, G., Sahu, S.K.: Astrophys. Space Sci. 288, 509 (2003)

[10] Mohanty, G., Sahu, S.K.: Astrophys. Space Sci. 291, 75 (2004)

[11] Tripathi, S.K., Nayak, S.K., Sahu, S.K., Routray, T.R.: Int. J. Theor. Phys. 48, 213 (2009)

[12] Reddy, D.R.K., Naidu, R.L., Rao, V.U.M.: Astrophys. Space Sci. 306, 185 (2006)

[13] Reddy, D.R.K., Govinda, P., Naidu, R.L.: Int. J. Theor. Phys. 47, 2966 (2008)

[14] Reddy, D.R.K., Naidu, R.L.: Astrophys. Space Sci. 312, 99 (2007)

[15] Rao, V.U.M., Vinutha, T., Shanthi, M.V., Kumari, G.S.D.: Astrophys. Space Sci. 317, 89 (2008)

[16] Rao, V.U.M., Shanthi, M.V., Vinutha, T.: Astrophys. Space Sci. 317, 27 (2008)

[17] Rao, V.M.U., Kumari, G.S.D., Sireesha, K.V.S.: Astrophys. Space Sci. 335, 635 (2011)

[18] Adhav, K.S., Ugale, M.R., Kale, C.B., Bhende, M.P.: Int. J. Theor. Phys. 46, 3122 (2007)

[19] Katore, S.D., Adhav, K.S., Shaikh, A.Y., Sarkate, N.K.: Int. J. Theor. Phys. 49, 2358 (2010)

[20] Sahu, S.K. J. Mod. Phys. 1, 67 (2010)

[21] Singh, C.P.: Braz. J. Phys. 39, 619 (2009)

[22] Pradhan, A., Singh, S.K.: Elect. J. Theor. Phys. 7, 407 (2010)

[23] Naidu, R.L., Satyanarayana, B., Reddy, D.R.K.: Astrophys. Space Sci. 338, 333 (2012)

[24] Reddy, D.R.K., Satyanarayana, B., Naidu, R.L., Astrophys. Space Sci. DOI 10.1007/s10509-012-1007-8 (2012)

[25] Kumar, S., Singh, C.P.: Int. J. Theor. Phys. 47, 1722 (2008)

[26] Riess, A.G., et al. (Supernova Search Team Collaboration): Astron. J. 116, 1009 (1998)

[27] Perlmutter, S., et al. (Supernova Cosmology Project Collaboration): Nature 391, 51 (1998)

[28] Bennett, et al.: Astrophys. J. Suppl. 184, 1 (2003) 
[29] Tegmark et al.: Phys. Rev. D 69, 103509 (2004)

[30] Allen, S.W., et al.: Mon. Not. Roy. Astron. Soc. 353, 457 (2004)

[31] Tonry, J.L., et al. (Supernova Search Team Collaboration): Astrophys. J. 594, 1 (2003)

[32] Riess, A.G., et al. (Supernova Search Team Collaboration): Astrophys. J. 607, 665 (2004)

[33] Clocchiatti, A., et al. (High Z SN Search Collaboration): Astrophys. J. 642, 1 (2006)

[34] Padmanabhan, T., Roychowdhury, T.: Mon. Not. R. Astron. Soc. 344, 823 (2003)

[35] Amendola, L.: Mon. Not. R. Astron. Soc. 342, 221 (2003)

[36] Riess, A.G., et al.: Astrophys. J. 560, 49 (2001)

[37] Socorro, J., Sabido, M., Sánchez, G.M.A., Frías Palos M.G.: Revista Mexicana De Fisica 56, 166 (2010)

[38] Jamil, M., Ali, S., Momeni, D., Myrzakulov, R.: arXiv:1201.0895[physics.gen-ph] (2012)

[39] Pradhan, A., Dubey, A.S., Khare, R.K.: Rom. J. Phys. 57, (2012) (to appear)

[40] Saha, B., Amirhashchi, H., Pradhan, A.: arXiv:1108.2133 [gr-qc] (2011)

[41] Pradhan, A., Amirhashchi, H.: Mod. Phys. Lett. A 28, 2261 (2011)

[42] Amirhashchi, H., Pradhan, A., Saha, B.: Chin. Phys. Lett. 28, 039801 (2011)

[43] Yadav, A.K.: arXiv:1202.3644[physics.gen-ph] (2012)

[44] Collins, C.B.: J. Math. Phys. 18, 2116 (1977)

[45] Schuecker, R., et al.: Astrophys. J. 496, 635 (1998)

[46] Cunha, C.E., Lima, M., Ogaizu, H., Frieman, J., Lin, H.: Mon. Not. Roy. Astron. Soc. 396, 2379 (2009)

[47] MacCallum, M.A.H.: Commun. Math. Phys. 20, 57 (1971)

[48] Caldwell, R.R., Komp, W., Parker, L., Vanzella, D.A.T.: Phys. Rev. D 37, 023513 (2006) 